\title{
Possible Protective Role of Melatonin in Pediatric Infectious Diseases and Neurodevelopmental Pathologies
}

\author{
Antonio Molina-Carballo ${ }^{1}$ Antonio Emilio Jerez-Calero ${ }^{1}$ Antonio Muñoz-Hoyos ${ }^{1}$ \\ ${ }^{1}$ Department of Pediatrics, Unit of Pediatric Neurology and \\ Neurodevelopment, Clínico San Cecilio University Hospital, the \\ Andalusian Health Service, School of Medicine, University of \\ Granada, Granada, Spain \\ Address for correspondence Antonio Molina-Carballo, MD, \\ Neuropediatrician, UGC Pediatría, Hospital Clínico San Cecilio, \\ Avda del Conocimiento sn, 18016-Granada, Spain \\ (e-mail: amolinac@ugr.es).
}

J Child Sci 2020;10:104-109.
Abstract
Keywords
- melatonin
- inflammatory disorders
- COVID-19
- neurodevelopmental pathologies
- children

Melatonin, produced in every cell that possesses mitochondria, acts as an endogenous free radical scavenger, and improves energetic metabolism and immune function, by complex molecular crosstalk with other intracellular compounds. There is greatly increasing evidence regarding beneficial effects of acute and chronic administration of high melatonin doses, in infectious, developmental, and degenerative pathologies, as an endothelial cell and every cell protectant.

\section{Introduction}

Melatonin, that may be produced in every cell that possesses mitochondria, ${ }^{1}$ acts as an endogenous free radical scavenger, and improves energetic metabolism and immune function by complex molecular crosstalk with other intracellular compounds. ${ }^{2}$ There is increasing evidence regarding the beneficial effects of acute and chronic administration of high melatonin doses in infectious and degenerative pathologies as a protectant of all cells, including endothelial cells.

To slow the stealthy spread of the new coronavirus disease 2019 (COVID-19) pandemic, until the development of a vaccine, we need not only effective treatments but additional strategies to prevent and/or ameliorate the infection reinforcing the immune capacity of the host. In this way, several authors have advocated for the use of high oral doses of the neurohormone melatonin. ${ }^{3,4}$ COVID-19 is caused by a positive-sense ribonucleic acid virus named the severe acute respiratory syndrome coronavirus 2 (SARS-CoV-2), which is associated with mortality rates that can exceed $6 \%$. Older patients with comorbid chronic conditions have been associ- ated with even higher mortality rates. In children, the clinical, laboratory, and radiologic features are similar for all coronavirus types. ${ }^{5}$

The COVID-19 virus has been shown to cause oxidative damage and cellular and organ failure by disabling the immune system, enhancing blood coagulation, and inducing a marked inflammatory response with generation of free radicals. COVID-19-endothelial activation could explain the systemic impaired microcirculatory function in different vascular beds and their clinical sequelae. ${ }^{6,7}$ Those sequelae are similar to that reported in Ebola virus patients. ${ }^{4}$ Angiotensin-converting enzyme 2 (ACE2) is the receptor that SARS-CoV-2 uses for penetration into the cells. ${ }^{8}$ SARS-CoV-2 exacerbation of animal lung injury by decreasing ACE2 is reversed by angiotensin receptor blockers (ARBs) and ACE inhibitors (ACEI) have been shown to induce upregulation of ACE2 expression. ${ }^{9}$ ACE2 has a broad expression pattern in the human body with strong expression in several organs, including in type II alveolar cells in the lungs. ACE2 primarily acts to counterbalance the effect of ACE; as ACE generates angiotensin II from angiotensin I, ACE2 generates angiotensin (1-7) from angiotensin II which shifts received

June 6, 2020

accepted

August 7, 2020
DOI https://doi.org/

10.1055/s-0040-1716713. ISSN 2474-5871.
Copyright @ 2020 Georg Thieme Verlag License terms

KG Stuttgart · New York

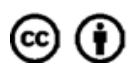


the balance from vasoconstriction to vasodilation in the vascular bed. ${ }^{10}$ Several studies recently published shows that neither ARBs nor ACEI, modifies the clinical course of COVID-19 infection. ${ }^{11}$

\section{Options for COVID-19 Treatment}

In addition to antivirals and drugs to treat the inflammatory cytokine storm, it appears that therapies aimed at protecting the endothelium may play a role. ${ }^{7,12}$ Several lines of evidence reinforce the hypothesis of utility of melatonin preventing endotheliitis, and as pleiotropic cytoprotectant at high oral doses. ${ }^{13,14}$ Melatonin may reduce viral infections based on its wide ranging effects as a potent antioxidant, ${ }^{15}$ pollutants protector, ${ }^{16}$ and anti-inflammatory agent ${ }^{17}$ in addition to its direct modulation of intracellular and extracellular processes which is associated with improvements in energy metabolism and immune function. ${ }^{15}$

There seems to be a few cellular functions that are not impacted by melatonin, which in high concentrations is rapidly taken up by the mitochondria. The mitochondria synthetizes high rates of melatonin that do not reach the interstitial nor vascular spaces. In this way, several studies have shown that melatonin has a protective role in human infections, such as viral encephalitis and severe infant bronchiolitis. ${ }^{18}$

\section{Mechanism of Melatonin Actions}

The central circadian clock located in the suprachiasmatic nucleus $(\mathrm{SCN})$ receives direct retinal innervation to ensure its synchronization to day-night cycles. Further, the SCN coordinates all peripheral clocks, which, in turn, coordinates local physiologic functions with patterns of activity and/or feeding. The peripheral clocks direct local programs of circadian gene expression that regulate physiological rhythms critical to health. ${ }^{19}$ The production of the melatonin hormone by the pineal gland during nighttime senses the light/dark cycles contributing to the coordination of peripheral circadian rhythms. Functional circadian molecular clockwork develops in the late fetal and early postnatal period. During the early postnatal period, oscillations are entrained by nutritional cues. $^{20}$ Feeding schedules and exercise can also entrain central and peripheral clocks. Circadian rhythms are key regulators of immune function, metabolism, reproduction, and stem cell development. ${ }^{20}$

Chronodisruptors are exogenous and endogenous exposures or effectors which are chronobiologically active and can thus disrupt the timing and order. ${ }^{21}$ Chronodisruptors turn off the clock control of the mitochondrial function, leading to a bioenergetic decay and formation of reactive oxygen species that, in turn, activate the inflammasome. Chronodisruption is associated with alterations of the immune system, immunosenescence, impairment of energy metabolism, and reduction of pineal and extrapineal melatonin production. Under inflammatory conditions, several cytokines may promote chronodisruption leading to a major immune response. ${ }^{22}$ The broken clock is recovered by melatonin administration. ${ }^{22}$
Melatonin is synthesized from the essential aminoacid tryptophan, and 3 to $10 \%$ of tryptophan metabolism produces serotonin and melatonin. In addition to protein synthesis, $90 \%$ of tryptophan metabolism follows the oxidation by indoleamine-2,3- dioxygenase (IDO) resulting in the formation of kynurenine and metabolites. ${ }^{23}$

Pineal melatonin induces the circadian gene Bmal1, which disinhibits the pyruvate dehydrogenase complex (PDC), allowing mitochondrial conversion of pyruvate to acetyl-coenzyme A (acetyl-CoA), with result of increased the tricarboxylic acid cycle, oxidative phosphorylation, and adenosine triphosphate (ATP) production. ${ }^{24}$ This metabolic route is blocked by viral inhibition of Bmal1/PDC preventing the circadian "resetting" of mitochondrial metabolism exerted by pineal melatonin. Acetyl-CoA is a cosubstrate for arylalkylamine $\mathrm{N}$-acetyltransferase (NAT), the enzyme that initiates the melatonergic pathway by providing an acetyl group to serotonin. Consequently, pineal melatonin regulates mitochondrial melatonin and immune cell phenotype. Virus- and cytokine-storm-driven control of the pineal and mitochondrial melatonergic pathway therefore regulates mitochondrial and immune/glia responses, besides an increased gut permeability and disbiosis mediated by decreased levels of the short-chain fatty acids, such as butyrate, and increases circulating lipopolysaccharide (LPS). ${ }^{4,13}$ The end result is greater viral replication and host symptom severity. The counteracting effects of butyrate are partly mediated via an increase in the mitochondrial melatonergic pathway, showing interactions of the gut microbiome with melatonin. ${ }^{25}$

Early life biological stress and psychological stress induces IDO, the enzyme that initiates the kynurenine pathway (KP) of tryptophan metabolism with overproduction of immunosuppressive catabolites. ${ }^{26}$ Stress and infections change the composition of the gut microbiota, with a bidirectional microbial-neuroendocrine relationship, regulating the postnatal development and maturation of the immune and endocrine systems. ${ }^{27}$ Endogenous (KP) and bacterial (indole) tryptophan catabolites, acting by activation of aryl hydrocarbon receptor (AhR, a transcription factor that regulates gene expression), changes the regulation of intestinal immunity. ${ }^{28}$

Activation of KP metabolism by stress has been associated with several proinflammatory states including depression and other neuropsychiatric disorders. ${ }^{29,30}$ Ambient pollution may contribute to such proinflammatory states with higher incidence and severity of COVID-19, by two mechanisms: (1) The atmosphere, rich of air pollutants, may promote a longer permanence of the of viral particles in the air. ${ }^{31}$ (2) The exposure to polycyclic aromatic hydrocarbons (PAHs), that may induce impaired immune function and low-grade inflammation, thereby promoting a higher incidence and severity of the cytokine storm triggered by viral infections. In this sense, elevated expression of AhR and NLRP3 (NLR family pyrin domain containing-3) link PAH exposure to cytokine storm in healthy preschool children, both mediators with differential pattern of elevated levels of several interleukins (ILs), as well as tumor necrosis factor- $\alpha$, and interferon- $\gamma$ levels. ${ }^{32}$ Melatonin suppresses the NLRP3 inflammasome through the regulation 
of cellular redox status and the nuclear factor-KB (NF-KB) pathway. ${ }^{33}$ Melatonin exerts potent antioxidant properties due to its high bioavailability through the penetration of the blood-brain barrier and placenta. Indirectly, the antioxidant properties of melatonin are linked to an increased activity of superoxide dismutase, glutathione peroxidase, reductase, and catalase. $^{34}$

\section{Immunological Functions of Melatonin}

The immune modulator properties of melatonin ${ }^{35}$ displays both pro- and anti-inflammatory effects ${ }^{36}$ by both melatonin receptor-dependent and -independent mechanisms. ${ }^{37}$ Proinflammatory actions enhance the resistance against pathogens. ${ }^{4}$ In human colostrum, melatonin produced by immune-competent cells acts in an autocrine manner, enhancing the clearance of pathogens by increasing phagocyte efficiency. ${ }^{38}$ Melatonin reduces chronic and acute inflammation ${ }^{39}$ by modulation of both pro- and anti-inflammatory cytokines. ${ }^{36}$ In the cytoplasm, melatonin blocks the Ripk3 cascade, Drp1 activation, and cytochrome $\mathrm{c}$ release caused by external insults, leading to the cell receiving prosurvival signals. In mitochondria, melatonin modulates mitochondrial permeability transition pore opening and counteracts oxidative stress. ${ }^{36}$ In experimental studies, melatonin inhibits nitric oxide (NO) production induced by bacterial LPS which in turn activates the immune response dependent on the activation of NF-kB. ${ }^{40}$

As a summary of cell sites which exert its anti-inflammatory activity, melatonin inhibits: (1) the expression of inflammasome genes, including NLRP3, ASC, and thereby caspase- 1 and IL-1 $\beta$; (2) inflammasome activation, ${ }^{41}$ thus inhibiting caspase- 1 activation, cytokine release, and inhibition of pyroptosis, a proinflammatory form of cells ${ }^{42}$; (3) NF$\mathrm{KB}$ traslocation, ${ }^{43}$ with subsequent inhibition of cyclooxygenase and inducible NO synthase expression and reduces the production of prostanoids, leukotrienes, and NO, as other inflammatory mediators, such as chemokines and adhesion molecules ${ }^{40}$; and (4) NF-KB expression. ${ }^{17}$ All of these antiinflammatory mechanisms convey into immunoregulator, antipyretic, antioxidant, and endoplasmic reticulum stress modulator during viral infections. Consequently, melatonin enhances protective mechanisms for viral encephalitis ${ }^{44}$ and respiratory tract disorders. ${ }^{18}$

The dual roles of melatonin as both a pro- and antiinflammatory regulator that determines whether melatonin promotes one or the other mode of immunological response is not completely understood. The anti-inflammatory actions of melatonin are mostly prevailing with the important exception of autoimmune diseases. ${ }^{40}$

\section{Melatonin Potential Utility}

From the perinatal period, it has been verified that melatonin plays an important role in the antioxidant response. ${ }^{45}$ Melatonin scavenges free radicals, ${ }^{46}$ stimulates antioxidant enzymes, ${ }^{15}$ stimulates the immune system, ${ }^{17}$ has anti-inflammatory actions, ${ }^{36}$ and impacts platelet physiology and thrombin formation. ${ }^{47}$ Melatonin could lower the severity of
COVID-19 and other viral infections by interfering with the processes that promote blood coagulation and vascular inflammation, preventing damage to the endothelium with reduction of the extravasation of blood through the capillary walls. ${ }^{15}$ Additionally, melatonin's actions on platelet aggregation and thrombin production reduces blood coagulation.

Cytoprotection by melatonin is based on almost every cell in the body having mitochondria that produces melatonin, and the intracellular concentrations of melatonin are much higher than those circulating in blood. ${ }^{48}$ Intracellular melatonin does not reach the extracellular space and doses of melatonin much higher than those employed as a chronobiotic are needed to modify its intracellular levels. ${ }^{14}$

Neuroprotective properties of melatonin have been postulated for a long time in acute neurological pathologies such as ischemic stroke, ${ }^{49}$ which showed an impairment of nocturnal urinary melatonin excretion, associated with immunological changes. ${ }^{50}$ Recently, these neuroprotective properties have been demonstrated in all phases of hypoxic-ischemic encephalopathy of the newborn. ${ }^{43,51}$

As noted before, high circulating melatonin levels at night allows cells to switch from cytosolic glycolysis to mitochondrial glucose oxidation and oxidative phosphorylation for ATP production, which is associated with higher synthesis of acetyl-CoA. In the mitochondria, one of the functions of acetyl-CoA is a cofactor for the rate limiting enzyme, arylalkylamine NAT, in mitochondrial melatonin synthesis. The abilities of melatonin facilitating mitochondrial glucose oxidation and oxidative phosphorylation may inhibit cytokine storm induced by COVID-19. ${ }^{52}$

\section{Melatonin Properties and Suggested Therapeutic Doses}

Melatonin is easily synthesized, inexpensive, and has a very high safety profile with no toxic dose. ${ }^{14}$ The sleep-promoting dose, that is, 1 to $5 \mathrm{mg}$ daily, do no reach the intracellular space at sufficient concentrations to protect against serious pathologies and infections such as COVID-19. ${ }^{14}$ For this reason, Reiter et al have suggested a daily oral dose of at least $40 \mathrm{mg}$ of melatonin in adults, ${ }^{53}$ a dose that the authors have been taking and recommending for prophylactic use. Reiter et al also stated that "as there is no time or clinical trials to test the efficacy of melatonin at different concentrations, we suggest the use of melatonin (100 or $400 \mathrm{mg}$ per day) as an adjunct, especially if no efficient direct anti-viral treatment is available." 53 Currently in Spain, due to the magnitude of infected health care workers, a clinical trial in the prophylaxis of SARS-CoV-2 infection has been started in eight hospitals with 450 health care workers. ${ }^{54}$ Another aspect to consider is the dose in children, as it seems appropriate to us that at least during this pandemic stage, doses between 5 and $10 \mathrm{mg}$ depending on age could be useful.

In preterm infants, because the pharmacokinetic profile of melatonin differs from that of adults, oral doses of 0.5 to $5 \mathrm{mg} / \mathrm{kg}$, repeated every 12 to 24 hours, may obtain and maintain high serum concentrations. ${ }^{55}$ In newborns at term, melatonin also has a good safety profile. ${ }^{51,56}$ 


\section{Melatonin Utility in Pediatrics}

For COVID-19 infection in children, the only treatment recommendation suggests the use of nebulized interferon $\alpha-2 b$ and oral lopinavir/ritonavir together with corticosteroids for complications (acute respiratory distress syndrome, encephalitis, hemophagocytic syndrome, or septic shock) and intravenous immunoglobulin for severe cases. ${ }^{5}$

The ACE2 receptor that allows SARS-CoV-2 entry into cells was widely spread in specific cell types of maternal-fetal interface and fetal organs. ${ }^{6}$ High maternal morbidity and mortality was associated with SARS-CoV-2 during pregnancy, ${ }^{57}$ with a higher incidence of spontaneous miscarriage, premature delivery, and intrauterine growth restriction. ${ }^{58}$

Fetal or neonatal exposure to stress conditions, besides to impact melatoninergic system, also can impact the reninangiotensin-aldosterone system (RAAS), contributing to the establishment of persistent hemodynamic and structural alterations. Preterm birth may imply early exposure to that ex utero environment, altering organogenesis and maturation in the newborn. RAAS may be activated also as a consequence of kidney and heart immaturity to promote adaptive responses, increasing the risk of future cardiovascular diseases. In this sense, inflammatory cytokines may exert an in utero influence on later infant adiposity. ${ }^{59}$ In women with severe preeclampsia, melatonin has been shown to mitigate maternal endothelial pro-oxidant injury and could extend pregnancy duration with improved clinical outcomes. ${ }^{12}$

In newborns suffering from septic shock, high oral doses of melatonin reduced mortality, ${ }^{56}$ which was probably related to maintaining endothelial function altered by bacterial LPS in a similar way that vascular damage is induced by COVID-19.

Melatonin has protective activity in all phases of hypoxicischemic encephalopathy including attenuation of tertiary brain damage, ameliorating long-term negative outcomes. ${ }^{43}$ Hypothermia plus melatonin, administered intravenously at a neuroprotective dose of $5 \mathrm{mg} / \mathrm{kg} /$ day for 3 days, was shown to improve long-term neurodevelopment in asphyctic neonates born at term. ${ }^{51}$

Melatonin also may be useful in Kawasaki disease (KD) as an acute, febrile vasculitis affecting children of younger than 5 years of age, specifically in an uncommon subgroup of KD children that presented with shock and more severe laboratory markers of inflammation including increased levels of several cytokines. KD shock syndrome due to cytokine storm can lead to significant sequelae and poor outcomes. ${ }^{60}$

A set of relevant features has been selected (lymphopenia, blood sedimentation rate, C-reactive protein, ferritin, ILs, dimer D, procalcitonin), ${ }^{61}$ to implement a computational model to test how melatonin affect to COVID-19 in children. This computational model can be an artificial neural network or a deep learning approach according to the kind and nature of real data.

\section{Neurodevelopmental Disorders}

Maternal melatonin provides photoperiodic information to the fetus, influencing their regulation and timing internal rhythms and preparation for extrauterine development.
The absence of maternal pineal melatonin (i.e., premature birth) might determine abnormal brain programming of the fetus, with increased risk of cardiovascular, renal, endocrine, neurodevelopmental, cognitive, and behavioral impairment. ${ }^{62,63}$ Fetal growth restriction (FGR; which is the most common cause of placental insufficiency and are diagnosed in up to $9 \%$ of pregnancies) face with those increased risks and have been associated with low maternal melatonin levels. ${ }^{64}$ Whether prenatal stress induces FGR or not, the effect of prenatal environmental adversity on child neurodevelopmental delay seems to be mediated by higher levels of maternal inflammation, especially when persisting throughout pregnancy. ${ }^{30}$ Consequently, several clinical studies are ongoing to ameliorate outcomes in FGR using high oral doses of melatonin. ${ }^{64}$

\section{Conclusion}

The evidence provided by the literature, with mechanisms of action capable of modifying the pathophysiology of several developmental and inflammatory disorders, suggests that melatonin may be a good resource to ameliorate them. Nevertheless, melatonin has not been used to treat COVID19 nor other inflammatory pathologies, and therefore, should not be considered to be an effective treatment until fulfillment of randomized clinical trials.

Conflict of Interest

None declared.

\section{References}

1 Suofu Y, Li W, Jean-Alphonse FG, et al. Dual role of mitochondria in producing melatonin and driving GPCR signaling to block cytochrome c release. Proc Natl Acad Sci USA 2017;114(38): E7997-E8006

2 Zhao D, Yu Y, Shen Y, et al. Melatonin synthesis and function: evolutionary history in animals and plants. Front Endocrinol (Lausanne) 2019;10:249-249

3 Shneider A, Kudriavtsev A, Vakhrusheva A. Can melatonin reduce the severity of COVID-19 pandemic? Int Rev Immunol 2020;39 (04):153-162

4 Reiter RJ, Ma Q, Sharma R. Treatment of Ebola and other infectious diseases: melatonin "goes viral." Melatonin Res 2020;3:43-57

5 Chen ZM, Fu JF, Shu Q et al. Diagnosis and treatment recommendations for pediatric respiratory infection caused by the 2019 novel coronavirus. World J Pediatr 2020;16(03):240-246

6 Li M, Chen L, Zhang J, Xiong C, Li X. The SARS-CoV-2 receptor ACE2 expression of maternal-fetal interface and fetal organs by singlecell transcriptome study. PLoS One 2020;15(04):e0230295

7 Varga Z, Flammer AJ, Steiger P, et al. Endothelial cell infection and endotheliitis in COVID-19. Lancet 2020;395(10234):1417-1418

8 Zhou P, Yang X-L, Wang X-G, et al. A pneumonia outbreak associated with a new coronavirus of probable bat origin. Nature 2020;579(7798):270-273

9 Kuba K, Imai Y, Rao S, et al. A crucial role of angiotensin converting enzyme 2 (ACE2) in SARS coronavirus-induced lung injury. Nat Med 2005;11(08):875-879

$10 \mathrm{Kai} \mathrm{H}$, Kai M. Interactions of coronaviruses with ACE2, angiotensin II, and RAS inhibitors-lessons from available evidence and insights into COVID-19. Hypertens Res 2020;43(07):648-654

11 Jarcho JA, Ingelfinger JR, Hamel MB, D'Agostino RB Sr, Harrington DP. Inhibitors of the renin-angiotensin-aldosterone system and Covid-19. N Engl J Med 2020;382(25):2462-2464 
12 Hobson SR, Gurusinghe S, Lim R, et al. Melatonin improves endothelial function in vitro and prolongs pregnancy in women with early-onset preeclampsia. J Pineal Res 2018;65(03):e12508

13 Anderson G, Reiter RJ. Melatonin: roles in influenza, Covid-19, and other viral infections. Rev Med Virol 2020;30(03):e2109

14 Cardinali DP. Are melatonin doses employed clinically adequate for melatonin-induced cytoprotection? Melatonin Res 2019;2:106-132

15 Tan DX, Reiter RJ. Mitochondria: the birth place, battle ground and the site of melatonin metabolism in cells. Melatonin Res 2019;2:44-66

16 Xin Z, Zhang X, Hu W, et al. The protective effects of melatonin on organisms against the environmental pollutants of heavy metal and non-mental toxins. Melatonin Res 2019;2:99-120

17 Carrillo-Vico A, Lardone PJ, Alvarez-Sánchez N, Rodríguez-Rodríguez A, Guerrero JM. Melatonin: buffering the immune system. Int J Mol Sci 2013;14(04):8638-8683

18 Banerjee A, Czinn SJ, Reiter RJ, Blanchard TG. Crosstalk between endoplasmic reticulum stress and anti-viral activities: a novel therapeutic target for COVID-19. Life Sci 2020;255:117842

19 Hastings MH, Maywood ES, Brancaccio M. The mammalian circadian timing system and the suprachiasmatic nucleus as its pacemaker. Biology (Basel) 2019;8(01):E13

20 Logan RW, McClung CA. Rhythms of life: circadian disruption and brain disorders across the lifespan. Nat Rev Neurosci 2019;20(01): 49-65

21 Erren TC, Reiter RJ. Defining chronodisruption. J Pineal Res 2009; 46(03):245-247

22 Acuña-Castroviejo D, Rahim I, Acuña-Fernández C, et al. Melatonin, clock genes and mitochondria in sepsis. Cell Mol Life Sci 2017; 74(21):3965-3987

23 Stone TW. Tryptophan and kynurenines: continuing to court controversy. Clin Sci (Lond) 2016;130(15):1335-1337

24 Reiter RJ, Tan DX, Rosales-Corral S, Galano A, Jou M-J, AcunaCastroviejo D. Melatonin mitigates mitochondrial meltdown: interactions with SIRT3. Int J Mol Sci 2018;19(08):24-39

25 Ivanov DO, Evsyukova II, Mazzoccoli G, et al. The role of prenatal melatonin in the regulation of childhood obesity. Biology (Basel) 2020;9(04):E72

26 Notarangelo FM, Pocivavsek A. Elevated kynurenine pathway metabolism during neurodevelopment: implications for brain and behavior. Neuropharmacology 2017;112(Pt B):275-285

27 Julio-Pieper M, O'Connor RM, Dinan TG, Cryan JF. Regulation of the brain-gut axis by group III metabotropic glutamate receptors. Eur J Pharmacol 2013;698(1-3):19-30

$28 \mathrm{Gao}$ J, Xu K, Liu H, et al. Impact of the gut microbiota on intestinal immunity mediated by tryptophan metabolism. Front Cell Infect Microbiol 2018;8:13

29 Erny D, Hrabě de Angelis AL, Jaitin D, et al. Host microbiota constantly control maturation and function of microglia in the CNS. Nat Neurosci 2015;18(07):965-977

30 Girchenko P, Lahti-Pulkkinen M, Heinonen K, et al. Persistently high levels of maternal antenatal inflammation are associated with and mediate the effect of prenatal environmental adversities on neurodevelopmental delay in the offspring. Biol Psychiatry 2020;87(10):898-907

31 Frontera A, Martin C, Vlachos K, Sgubin G. Regional air pollution persistence links to COVID-19 infection zoning. J Infect 2020;81 (02):318-356

32 Cheng Z, Huo X, Dai Y, Lu X, Hylkema MN, Xu X. Elevated expression of AhR and NLRP3 link polycyclic aromatic hydrocarbon exposure to cytokine storm in preschool children. Environ Int 2020;139:105720

33 Moloudizargari M, Moradkhani F, Asghari N, et al. NLRP inflammasome as a key role player in the pathogenesis of environmental toxicants. Life Sci 2019;231:116585

34 Korkmaz A, Reiter RJ, Topal T, Manchester LC, Oter S, Tan DX. Melatonin: an established antioxidant worthy of use in clinical trials. Mol Med 2009;15(1-2):43-50
35 Mortezaee K, Potes Y, Mirtavoos-Mahyari H, et al. Boosting immune system against cancer by melatonin: a mechanistic viewpoint. Life Sci 2019;238:116960

36 Tarocco A, Caroccia N, Morciano G, et al. Melatonin as a master regulator of cell death and inflammation: molecular mechanisms and clinical implications for newborn care. Cell Death Dis 2019;10 (04):317

37 Cardinali DP. Melatonin: clinical perspectives in neurodegeneration. Front Endocrinol (Lausanne) 2019;10:480

38 Pires-Lapa MA, Tamura EK, Salustiano EM, Markus RP. Melatonin synthesis in human colostrum mononuclear cells enhances dectin-1-mediated phagocytosis by mononuclear cells. J Pineal Res 2013;55(03):240-246

39 Esposito E, Cuzzocrea S. Antiinflammatory activity of melatonin in central nervous system. Curr Neuropharmacol 2010;8(03): 228-242

40 Hardeland R. Aging, melatonin, and the pro- and anti-inflammatory networks. Int J Mol Sci 2019;20(05):E1223

41 Cao Z, Fang Y, Lu Y, et al. Melatonin alleviates cadmium-induced liver injury by inhibiting the TXNIP-NLRP3 inflammasome. J Pineal Res 2017;62(03). Doi: 10.1111/jpi.12389

42 Liu Z, Gan L, Xu Y, et al. Melatonin alleviates inflammasomeinduced pyroptosis through inhibiting NF-KB/GSDMD signal in mice adipose tissue. J Pineal Res 2017;63(01). Doi: 10.1111/ jpi.12414

43 Cardinali DP. An assessment of melatonin's therapeutic value in the hypoxic-ischemic encephalopathy of the newborn. Front Synaptic Neurosci 2019;11:34

44 Silvestri M, Rossi GA. Melatonin: its possible role in the management of viral infections-a brief review. Ital J Pediatr 2013;39:61

45 Muñoz-Hoyos A, Bonillo-Perales A, Avila-Villegas R, et al. Melatonin levels during the first week of life and their relation with the antioxidant response in the perinatal period. Neonatology 2007; 92(03):209-216

46 Reiter RJ, Mayo JC, Tan DX, Sainz RM, Alatorre-Jimenez M, Qin L. Melatonin as an antioxidant: under promises but over delivers. J Pineal Res 2016;61(03):253-278

47 Kornblihtt LI, Finocchiaro L, Molinas FC. Inhibitory effect of melatonin on platelet activation induced by collagen and arachidonic acid. J Pineal Res 1993;14(04):184-191

48 Venegas C, García JA, Escames G, et al. Extrapineal melatonin: analysis of its subcellular distribution and daily fluctuations. J Pineal Res 2012;52(02):217-227

49 Fiorina P, Lattuada G, Ponari O, Silvestrini C, Dall'Aglio P. Impaired nocturnal melatonin excretion and changes of immunological status in ischaemic stroke patients. Lancet 1996;347(9002): 692-693

50 Fiorina P, Lattuada G, Silvestrini C, Ponari O, Dall'Aglio P. Disruption of nocturnal melatonin rhythm and immunological involvement in ischaemic stroke patients. Scand J Immunol 1999;50(02): 228-231

51 Jerez-Calero A, Salvatierra-Cuenca MT, Benitez-Feliponi Á, et al. Hypothermia plus melatonin in asphyctic newborns: a randomized-controlled pilot study. Pediatr Crit Care Med 2020;21(07): 647-655

52 Reiter RJ, Sharma R, Ma Q, Dominquez-Rodriguez A, Marik PE, Abreu-Gonzalez P. Melatonin inhibits COVID-19-induced cytokine storm by reversing aerobic glycolysis in immune cells: a mechanistic analysis. Med Drug Discov 2020;6:100044

53 Reiter RJ, Abreu-Gonzalez P, Marik PE, Dominguez-Rodriguez A. Therapeutic algorithm for use of melatonin in patients with COVID-19. Front Med (Lausanne) 2020;7:226

54 García IG, Rodriguez-Rubio M, Mariblanca AR, et al. A randomized multicenter clinical trial to evaluate the efficacy of melatonin in the prophylaxis of SARS-CoV-2 infection in high-risk contacts (MeCOVID Trial): a structured summary of a study protocol for a randomised controlled trial. Trials 2020;21(01):466 
55 Carloni S, Proietti F, Rocchi M, et al. Melatonin pharmacokinetics following oral administration in preterm neonates. Molecules 2017;22(12):E2115

56 Gitto E, Karbownik M, Reiter RJ, et al. Effects of melatonin treatment in septic newborns. Pediatr Res 2001;50(06): 756-760

57 Wong SF, Chow KM, de Swiet M. Severe acute respiratory syndrome and pregnancy. BJOG 2003;110(07):641-642

58 Chen H, Guo J, Wang C, et al. Clinical characteristics and intrauterine vertical transmission potential of COVID-19 infection in nine pregnant women: a retrospective review of medical records. Lancet 2020;395(10226):809-815

59 Donnelly JM, Lindsay K, Walsh JM, et al. Perinatal inflammation and childhood adiposity - a gender effect? J Matern Fetal Neonatal Med 2020;33(07):1203-1210

60 Li Y, Zheng Q, Zou L, et al. Kawasaki disease shock syndrome: clinical characteristics and possible use of IL-6, IL-10 and IFN- $\gamma$ as biomarkers for early recognition. Pediatr Rheumatol Online J 2019;17(01):1

61 Zimmermann P, Curtis N. Coronavirus infections in children including COVID-19: an overview of the epidemiology, clinical features, diagnosis, treatment and prevention options in children. Pediatr Infect Dis J 2020;39(05):355-368

62 Baschat AA. Neurodevelopment after fetal growth restriction. Fetal Diagn Ther 2014;36(02):136-142

63 Motta-Teixeira LC, Machado-Nils AV, Battagello DS, et al. The absence of maternal pineal melatonin rhythm during pregnancy and lactation impairs offspring physical growth, neurodevelopment, and behavior. Horm Behav 2018;105:146-156

64 Palmer KR, Mockler JC, Davies-Tuck ML, et al. Protect-me: a parallel-group, triple blinded, placebo-controlled randomised clinical trial protocol assessing antenatal maternal melatonin supplementation for fetal neuroprotection in early-onset fetal growth restriction. BMJ Open 2019;9(06):e028243 